\title{
Preparation for a forthcoming task is sufficient to produce subsequent shift costs
}

\author{
THOMAS KLEINSORGE and PATRICK D. GAJEWSKI \\ Universität Dortmund, Dortmund, Germany
}

\begin{abstract}
Shifting from one task to another is associated with significant costs. Recently, it has been questioned whether the mere preparation for a forthcoming task, without the task's actually being executed, is sufficient to establish a new task set that results in shift costs when the execution of a task other than the prepared task is required. In a go/no-go study, it is shown that the mere preparation for a task is sufficient to produce shift costs, but only under conditions that encourage participants to engage in advance preparation for a precued task despite the possibility that the execution of this task will not always be required, because of occasional no-go trials. In addition, considerable reductions of shift costs after go trials could be observed under these conditions. When such a motivating context was not provided, only negligible shift costs were observed after a no-go trial, indicating that no task-set configuration had taken place without the need to perform the task. Furthermore, under these conditions, prolonging the preparation interval resulted in reaction time benefits that were similar for task shifts and repetitions, again indicating that no active task-set configuration took place.
\end{abstract}

In a typical reaction time (RT) experiment, participants are instructed in a single task, and they perform this task repeatedly in a large number of trials. In such an experiment, measured RT reflects performance after participants have adopted an effective task set (Rogers \& Monsell, 1995) for the instructed task. Unfortunately, the processes by which the instruction is transformed into this effective task set are withdrawn from observation. Over the last decade, an experimental paradigm called task switching has gained considerable interest because it promises to open a window for the study of at least some of the processes by which humans prepare themselves for a particular task. The main principle of these experiments is to compare the repeated performance of a task (task repetitions) with the performance of the same task when another task has been performed in the trial before (task shifts). The usually observed increase of RTs in task-shift trials, as compared with task repetitions, the "shift cost," is often taken as a measure of the duration of task-set reconfiguration processes (see, e.g., Rogers \& Monsell, 1995; Rubinstein, Meyer, \& Evans, 2001). Thus, the measurement of shift costs promises to provide an important tool for studying the intentional control of behavior.

However, the assumption that shift costs index the duration of task-set reconfiguration has been questioned right

The research reported in this article was supported by Grant KI 1205/2-1 of the Deutsche Forschungsgemeinschaft. We thank Gero Szepannek, Kathrin Schumacher-Kalb, and Thomas Kohlborn for assistance in running the experiments and analyzing the data, and Stefan Lapp for providing the software. Correspondence should be addressed to T. Kleinsorge, Institut für Arbeitsphysiologie an der Universität Dortmund, Ardeystraße 67, D-44139 Dortmund, Germany (e-mail: kleinsorge@ifado.de). from the start of this research (see, e.g., Allport, Styles, \& Hsieh, 1994). One of the main findings that motivated this skepticism is the only partial reduction of shift costs when participants are given more time to prepare for a forthcoming task shift. Nevertheless, the fact that increasing preparation time reduces shift costs to a certain degree has been taken as evidence that at least this reduction of shift costs indexes processes of advance task reconfiguration (e.g., Meiran, Chorev, \& Sapir, 2000). Thus, the reasoning is that although residual shift costs-that is, the proportion of shift costs that remains after sufficient preparation time-may reflect processes extraneous to preparatory processes such as proactive interference (e.g., Waszak, Hommel, \& Allport, 2003), the proportion of shift costs that vanishes as a function of the length of a preparatory interval can nevertheless provide a useful measure of advance task-set reconfiguration.

To interpret a reduction of shift costs as a measure of advance task-set reconfiguration presupposes that advance task-set configuration indeed takes place during a preparatory interval. There is reason to believe that this may not always be the case. In most task-switching experiments, preparation for the next task is not necessary for performance of the task, because the imperative stimuli convey all the information that is needed. As De Jong (2000) has argued, advance preparation for a task requires (1) an explicit goal or intention to engage in preparatory activity, and (2) the retrieval of the action that is associated with this intention at the start of the preparation interval. Thus, whenever the basic activation level of the intention to engage in advance preparation is too low or the retrieval of the action that is associated with this intention fails, advance task-set reconfiguration is not to be expected (cf. De Jong, Berendsen, \& Cools, 1999). Importantly, holding 
an intention to engage in advance preparation at a high level of activation is likely to require substantial effort that in most task-switching experiments is rewarded by nothing more than an enhanced speed of responding. As a consequence, variables that affect participants' motivation to engage in advance preparation can be expected to have significant effects on the reduction of shift costs that can be observed with longer preparation intervals. This point will be taken up below.

If preparing for a new task results in at least a partial configuration of a task set suitable for the performance of the new task, then actually performing another task should be associated with a cost. That is, preparation for a task should result in costs when a task other than the prepared one has to be performed, to the extent that preparation results in a reconfigured task set. If, on the other hand, shifting away from a prepared (but not executed) task does not result in shift costs, it is questionable whether advance task-set reconfiguration in the sense of preparatory processes that are specific for a certain task have taken place at all. This, in turn, would further question the usefulness of shift costs as a measure of task-set reconfiguration.

Recently, Koch and coworkers (Koch \& Philipp, 2003; Schuch \& Koch, 2003) presented evidence that preparation for a new task does indeed result in no costs when actually another task has to be performed. In a series of experiments that aimed mainly at investigating the role of inhibitory processes as an origin of residual shift costs, they combined task switching with the go/no-go methodology and observed no shift costs when a task shift occurred after a no-go trial. That is, when participants prepared for but did not perform a particular task, it did not matter whether the task performed in the following go trial was the same as the one prepared for in the preceding nogo trial or different from this task. They interpreted their finding as indicating that response selection in a preceding trial is necessary for the occurrence of shift costs. When a task is merely prepared for but no response selection has taken place with that task, the corresponding task set is not configured to a degree that is sufficient to result in shift costs.

Koch and colleagues reported another observation which suggests that preparation does not result in the establishment of a new task set. In their experiments, the reduction of shift costs as a function of the length of the cue-target interval (CTI) - that is, the reduction of shift costs when more time for preparation was available - was quite small and sometimes statistically unreliable.

We reasoned that both findings, the absence of shift costs after no-go trials and the only small reduction of shift costs with long CTIs, might have been due not so much to structural constraints of preparatory processes but to peculiarities of the experimental paradigm. Specifically, the fact that participants experienced that preparation for a precued task was in vain in a substantial proportion of trials (the no-go trials) might have led them to refrain from engaging in advance task-set reconfiguration. Although participants responded faster with longer CTIs than with shorter CTIs, this might have been due to more unspecific preparation that affected shift trials only slightly more than task repetitions. If so, it would not be too surprising to find no shift costs after a no-go trial. However, the implication of this would not implicate structural constraints of advance task preparation without responding but rather point to the importance of taking into account motivational factors in accounting for shift costs. Thus, the theoretical focus would shift from an alleged limitation to prepare for a task without actually responding to the motivational boundary conditions under which advance task preparation is likely to occur.

In the experiment described below, we replicated the basic paradigm of Schuch and Koch (2003, Experiment 1) but added another condition intended to enhance participants' motivation to engage in advance preparation despite the occurrence of no-go trials. In this condition, participants were veridically informed that after a no-go trial, in $80 \%$ of the following go trials a task repetition would occur. This way, participants knew that preparing for the precued task would be beneficial even when a no-go trial occurred, because the next task would be the same as the task precued for the no-go trial with a high probability.

Of course, such a manipulation introduces biased expectancies regarding task repetitions after a no-go trial. Therefore, observing an effect only in trials that followed a no-go trial would provide rather ambiguous evidence. However, we expected that our experimental manipulation would enhance participants' general motivation to engage in advance preparation. This should be indicated in reduced shift costs also after go trials as well.

\section{METHOD}

\section{Participants}

Fifteen male and 9 female volunteers participated. They were paid for their service. Their mean age was 25.4 years (range, 21-32).

\section{Task, Apparatus, and Stimuli}

The participants had to respond according to the numerical magnitude (more or less than five) or the odd/evenness of a centrally presented digit $(1.0 \times 1.0 \mathrm{~cm})$. The current task was indicated by a cue that surrounded the position of the digit. The cue consisted of the outline of a diamond $5.0 \mathrm{~cm}$ tall $\times 6.5 \mathrm{~cm}$ wide, indicating the odd/even task, or of the outline of a square $(4.5 \times 4.5 \mathrm{~cm})$, indicating the magnitude task. The stimuli consisted of the digits 1-9, excluding 5 . They were presented in white on a black background, on a 14-in. VGA monitor.

Responses consisted of pressing one of two horizontally arranged keys with the index fingers of the left and right hand. "Small" and "even" responses were assigned to the left key, and "large" and "odd" responses were assigned to the right key.

High and low tones of 50-msec duration served as go and no-go signals, respectively.

\section{Design}

The experiment had a $2 \times 2 \times 2 \times 2$ design with the withinparticipants factors go in trial $n-1$ (go vs. no go), CTI (100 vs. $1,000 \mathrm{msec}$ ), and trial type (task shift vs. repetition), and the between-participants factor context (neutral, repetition expectancy). Note that the latter factor affected expectancies only after no-go trials, not after go trials. CTI alternated across blocks of trials. In each 
context group, 6 participants started with the long CTI and the remaining 6 participants started with the short CTI.

The task sequence was random, with the following restrictions. Each task occurred with equal frequency, and for each task, there were equal numbers of task shift and repetition trials. In addition, the response sequence was controlled up to trial $n-2$, which ensured that all response repetitions and response alternations would occur with equal frequency and would be preceded with equal frequency by response repetitions and alternations. The task sequences and response sequences were orthogonally combined. Twenty-five percent of the trials were randomly converted into no-go trials, with the restriction that (1) no no-go trial was preceded by another no-go trial and (2) for the repetition-expectancy group, $80 \%$ of the no-go trials were followed by a repetition of the task cued in the no-go trial.

\section{Procedure}

The participants were given written instructions that explained the tasks. The instructions for the two groups of participants differed only with respect to one paragraph added to the instruction for the repetition expectancy group. This paragraph explained that after a no-go trial, in the majority of cases, the task cued for that trial would be repeated in the following go trial, and that it would be helpful to stay in a prepared state for this task.

A trial started with the presentation of the cue. Depending on the condition, after 100 or $1,000 \mathrm{msec}$ an imperative stimulus (digit) was displayed inside the cue. Simultaneously with the digit, a high or a low tone was presented for $50 \mathrm{msec}$. The low tone served as the nogo signal. When the participant responded despite the presentation of a low tone, error feedback ("do not press any key," in German) was presented for $500 \mathrm{msec}$. When the participant correctly refrained from responding, the display was cleared after 1,000 $\mathrm{msec}$. In go trials, the stimulus stayed on the screen until the participant responded.

The response-stimulus interval (RSI), which included the CTI, was 1,600 msec. During the interval between the response of trial $n-1$ and the onset of the cue for trial $n$ (RSI - CTI), the screen was blank.

Each block consisted of 97 trials. The first trial was randomly chosen. The remaining trials resulted from a factorial combination of the factors task, previous task, go/no-go, response, response in trial $n-1$, and response in trial $n-2$. After each block of trials, mean RT of correct trials was displayed on the screen. There were 12 blocks of 97 trials.

\section{RESULTS}

The first two blocks were considered practice and were not analyzed. Prior to analyses, trials that followed an error $(5.0 \%)$ or in which RT was below $100 \mathrm{msec}$ or above
$2,500 \mathrm{msec}(0.2 \%)$ were discarded from the data set. The randomly chosen first trial of each block was also dropped. From the RT analysis error trials were excluded.

Because nothing in the error data indicated a speedaccuracy tradeoff (cf. Table 1), only the results of the RT analyses will be reported here.

Individual mean RTs were computed for each combination of the factors task, trial type, CTI, and go in trial $n-1$ and later collapsed over the task factor. Mean RTs were analyzed by means of a $2 \times 2 \times 2 \times 2$ analysis of variance (ANOVA) with repeated measurements on the factors trial type (task shift vs. repetition), CTI (100 vs. $1,000 \mathrm{msec}$ ), go in $n-1$ (yes vs. no), and the betweenparticipants factor context (neutral, repetition expectancy).

The variation of the context had a significant main effect $\left[F(1,22)=4.90, M S_{\mathrm{e}}=444,338, p<.05\right]$. The group with the neutral context had significantly $(p<.05)$ longer RTs $(M=938 \mathrm{msec})$ than did the group which expected a task repetition after a no-go trial $(M=788 \mathrm{msec})$. This finding provides initial evidence that the two contexts affected participants' motivation differently.

Prolonging the CTI from 100 to $1,000 \mathrm{msec}$ significantly reduced mean RTs from 939 to $787 \mathrm{msec}[F(1,22)=$ $\left.85.70, M S_{\mathrm{e}}=25,730, p<.001\right]$. This finding represents the general preparation effect. Task shifts went along with significantly slower responses $(M=921 \mathrm{msec})$ than those for task repetitions $(M=805 \mathrm{msec})[F(1,22)=72.04$, $\left.M S_{\mathrm{e}}=17,770, p<.001\right]$. After no-go trials, participants responded significantly slower $(M=894 \mathrm{msec})$ than after go trials $(M=832 \mathrm{msec})\left[F(1,22)=32.83, M S_{\mathrm{e}}=\right.$ $10,897, p<.0011] .^{1}$

The most important questions addressed by the present experiment relate to the interactions of CTI and trial type and of trial type and go in $n-1$ and their possible modulation by the context factor. The first-order interaction of CTI and trial type was significant $\left[F(1,22)=12.64, M S_{\mathrm{e}}=\right.$ $5,086, p<.01]$, as was the second-order interaction of context, CTI, and trial type $\left[F(1,22)=6.12, M S_{\mathrm{e}}=5,086\right.$, $p<.05]$. In the neutral context group, shift costs amounted to $114 \mathrm{msec}$ with a short CTI and were only slightly reduced to $98 \mathrm{msec}$ when the CTI was long. For the repetition expectancy group, shift costs were reduced from

Table 1

Mean Reaction Times (RTs) nd Error Rates (ERs) as a Function of CTI, Context, Trial Type, and Go/No-Go in $n-1$

\begin{tabular}{|c|c|c|c|c|c|c|c|c|}
\hline \multirow[b]{3}{*}{ Trial Type } & \multicolumn{4}{|c|}{ Neutral Context } & \multicolumn{4}{|c|}{ Repetition Expectancy } \\
\hline & \multicolumn{2}{|c|}{ Go in $n-1$} & \multicolumn{2}{|c|}{ No Go in $n-1$} & \multicolumn{2}{|c|}{ Go in $n-1$} & \multicolumn{2}{|c|}{ No Go in $n-1$} \\
\hline & RT & ER & RT & ER & RT & ER & RT & ER \\
\hline \multicolumn{9}{|c|}{ CTI 100} \\
\hline Shift & 1062 & 8.8 & 1064 & 5.4 & 955 & 8.1 & 959 & 6.3 \\
\hline Repetition & 878 & 3.8 & 1019 & 5.8 & 748 & 3.6 & 828 & 5.2 \\
\hline \multicolumn{9}{|c|}{ CTI 1000} \\
\hline Shift & 919 & 7.8 & 922 & 5.1 & 747 & 7.2 & 740 & 6.8 \\
\hline Repetition & 734 & 3.5 & 910 & 5.4 & 617 & 3.7 & 708 & 4.8 \\
\hline
\end{tabular}


$169 \mathrm{msec}$ with a short CTI to $81 \mathrm{msec}$ with a long CTI. These observations provide evidence that the participants in the repetition expectancy group were indeed generally more motivated to engage in advance task preparation than the participants in the neutral context group.

As for the interaction of trial type and go in $n-1$ and its possible modulation by the context factor, both the first-order interaction of trial type and go in $n-1[F(1,22)=$ $\left.64.42, M S_{\mathrm{e}}=5,632, p<.001\right]$ and the second-order interaction of context, trial type, and go in $n-1[F(1,22)=$ $\left.5.37, M S_{\mathrm{e}}=5,632, p<.05\right]$ were significant. In the neutral context group, shift costs amounted to $185 \mathrm{msec}$ after a go trial but only to a nonsignificant $27 \mathrm{msec}$ after a nogo trial. In the repetition expectancy group, shift costs amounted to $169 \mathrm{msec}$ after a go trial. After a no-go trial, shift costs amounted to $82 \mathrm{msec}$ ( $p<.01$, according to a Duncan post hoc test) in this group, demonstrating considerable shift costs after a no-go trial. As revealed by a one-way ANOVA, the amount of shift costs after a no-go trial differed significantly between the two context groups $\left[F(1,22)=4.85, M S_{\mathrm{e}}=4,223, p<.05\right]$. Thus, whereas in the neutral-context group no significant shift costs after no-go trials could be observed, in the repetition expectancy group substantial shift costs after no-go trials showed up. No other effects reached statistical significance.

\section{DISCUSSION}

The results of the experiment are clear-cut. First, with the neutral-context group, we were able to replicate the findings of Schuch and Koch (2003, Experiment 1): We observed no significant shift costs after a no-go trial, and the reduction of shift costs due to a longer CTI was quite small. Second, the repetition expectancy group produced clearly different results. In this group, we observed considerable shift costs both after go and after no-go trials. Furthermore, the reduction of shift costs due to a longer CTI was much more pronounced.

Our observation of significant shift costs following a no-go trial in the repetition expectancy group shows that preparation for a forthcoming task is sufficient to produce shift costs. This contrasts with Schuch and Koch's (2003) suggestion that response selection within a task is crucial to observing shift costs for shifting away from the prepared task. ${ }^{2}$ Our findings clearly demonstrate that observing shift costs after no-go trials is dependent on participants' strategies in dealing with the occurrence of no-go trials. If they are motivated to engage in advance preparation despite the possibility that the present trial turns out to be a no-go trial because they expect a repetition of the current task in that case, shift costs follow when actually another task has to be performed.

However, our observations for trials that followed a nogo trial are somewhat ambiguous, because the probability structure regarding the following trial differed between the two experimental groups. Therefore, the different observations regarding shift costs after no-go trials might be accounted for in terms of local strategies that affected only trials that followed a no-go trial. In contrast, our observation that participants in the repetition expectancy group were much more successful than participants in the neutralcontext group in reducing their shift costs after go trials when the CTI was long is not open to an explanation that rests only on differences between the two groups that are confined to different strategies in dealing with no-go trials. The fact that the repetition expectancy group showed clear indications of advance preparation for the precued task after a go trial, whereas the neutral-context group did not, provides clear evidence that the manipulation regarding the probability of task repetitions after no-go trials affected the way participants dealt with the experimental situation as a whole.

Our findings are in line with our initial suspicion that including no-go trials in a task-shifting experiment discourages participants from engaging in advance preparation for a precued task because this preparation turns out to be in vain in a substantial proportion of trials. This results not only in an absence of shift costs after no-go trials, but also in a lack of advance preparation for a new task, and therefore in only negligible variations of shift costs across different preparation intervals.

In the beginning of the present article, we outlined some of the critique raised against using shift costs as a measure of advance task-set reconfiguration. One line of critique is based on observations that a substantial proportion of shift costs seems to be immune against manipulations that aim at varying the amount of preparatory activity that takes place before an actual task shift. Although we are quite sure that a substantial proportion of shift costs does in fact reflect factors other than preparatory processes, the present findings show that merely providing participants with more time for preparation does not guarantee that they actually engage in task-specific preparatory activity. Thus, observing only modest (if any) reductions of shift costs despite large variations of the interval that is available for advance preparation does not in principle undermine the usefulness of shift-cost reductions as a measure of preparatory activity. It only emphasizes the importance of taking into account motivational factors that modulate the probability that participants really engage in advance preparation (cf. De Jong, 2000, for a similar argument). Such factors include the length of trial blocks (De Jong, 2000), the explicitness of task precues (De Jong et al., 1999), monetary rewards (Nieuwenhuis \& Monsell, 2002), and the validity of precues (Kleinsorge, Gajewski, \& Heuer, in press). The present study adds to this evidence and demonstrates that relatively subtle manipulations such as the introduction of no-go trials that aim at something completely different (the involvement of response selection processes) can have strong motivational effects that may mimic the effects of alleged structural constraints of task preparation. Therefore, not observing shift costs after no-go trials in such experiments does not necessarily demonstrate structural limitations of advance task-set reconfiguration, but merely strategically determined limitations of participants' willingness to engage in such preparatory activities. 


\section{REFERENCES}

Allport, D. A., Styles, E. A. \& Hsieh, S. (1994). Shifting attentional set: Exploring the dynamic control of tasks. In C. Umiltà \& M. Moscovitch (Eds.), Attention and performance XV: Conscious and nonconscious information processing (pp. 421-452). Cambridge, MA: MIT Press.

DE JONG, R. (2000). An intention-activation account of residual switch costs. In S. Monsell \& J. Driver (Eds.), Attention and performance XVIII: Control of cognitive processes (pp. 357-376). Cambridge, MA: MIT Press.

De Jong, R., Berendsen, E., \& Cools, R. (1999). Goal neglect and inhibitory limitations: Dissociable causes of interference effects in conflict situations. Acta Psychologica, 101, 379-394.

De Jong, R., Coles, M. G. H., \& Logan, G. D. (1995). Strategies and mechanisms in nonselective and selective inhibitory motor control. Journal of Experimental Psychology: Human Perception \& Performance, 21, 498-511.

Kleinsorge, T., GAJewski, P. D., \& Heuer, H. (in press). Task sets under reconstruction: Effects of partially incorrect precues. Quarterly Journal of Experimental Psychology.

KocH, I., \& PHILIPP, A. M. (2003). Effects of response selection on the task-repetition benefit in task switching. Manuscript submitted for publication.

MeIran, N., Chorev, Z., \& SAPIR, A. (2000). Component processes in task switching. Cognitive Psychology, 41, 211-253.

NieuWenhuis, S., \& MONSEll, S. (2002). Residual costs in task switching: Testing the failure-to-engage hypothesis. Psychonomic Bulletin \& Review, 9, 86-92.

Rogers, R. D., \& MONSELl, S. (1995). The costs of a predictable switch between simple cognitive tasks. Journal of Experimental Psychology: General, 124, 207-231.

Rubinstein, J., MEYER, D. E., \& EvAns, J. E. (2001). Executive control of cognitive processes in task switching. Journal of Experimental Psychology: Human Perception \& Performance, 27, 763-797.

SCHUCH, S., \& KoCH, I. (2003). The role of response selection for inhibition of task sets in task shifting. Journal of Experimental Psychology: Human Perception \& Performance, 29, 92-105.

WASZAK, F., Hommel, B., \& Allport, A. (2003). Task-switching and long-term priming: Role of episodic stimulus-task bindings in taskshift costs. Cognitive Psychology, 46, 361-413.

\section{NOTES}

1. Increased RTs after no-go trials seem to be a general phenomenon that has also been reported by Schuch and Koch (2003) and Koch and Philipp (2003). One possibility to account for this finding is to assume that after a no-go trial the decision not to respond is "primed," and that this finding carries over to the following go trial. Apart from such a more "cognitive" presetting not to respond, more peripherally operating mechanisms of withholding a response might be involved in no-go trials (cf. De Jong, Coles, \& Logan, 1995), which might affect the motor system's readiness in a following go trial.

2. One might argue that we cannot exclude that participants in the repetition expectancy group did actually engage in some kind of "covert" response selection in no-go trials in order to enhance their preparation for the task cued in the no-go trial. This was possible, because an imperative stimulus was presented in the no-go trials. In order to check for this possibility, we ran another group of participants under conditions that replicated those for the repetition expectancy group with the exception that no imperative stimuli were presented in no-go trials. We still observed significant shift costs after no-go trials.

(Manuscript received December 2, 2002; revision accepted for publication April 3, 2003.) 